\title{
A Storage Architecture for High Speed Signal Processing: Embedding RAID 0 on FPGA
}

\author{
Ningxiao Sun, Qiongzhi Wu, Zhaojian Jin
}

School of Information and Electronics, Beijing Institute of Technology, Beijing, China.

Email: sunningxiao@sina.com

Received April 20 $0^{\text {th }}, 2012$; revised May 25 $5^{\text {th }}, 2012$; accepted June $10^{\text {th }}, 2012$

\begin{abstract}
The article proposes a new architecture based on RAID 0 tech in computer science for signal processing field to store high speed data. It is composed of SSD driven by FPGA, called SSD-based RAID on FPGA. This new architecture features high storage rate, mass capacity and small volume, and it is an efficient solution to store high speed data. The article describes the construction of SRF in details, and shows the test result of a demo system based on the architecture.
\end{abstract}

Keywords: RAID on FPGA; SSD; High Speed Data Storage

\section{Introduction}

In signal processing field, the system designer has been greatly forced to choose between real-time processing and the storage capacity of the acquisition signal. In the past, the latter always limits the maximum count rates supported without data loss. Systems which could perform both functions at the same time were usually too costly and complex. To solve these problems the first target is to find a sort of the storage medium [1].

Now, with recent advancements in the computer industry, the cost of NAND flash memory becomes lower and the density of NAND flash memory grows larger, the mass-production of solid state drives (SSD) based on NAND flash memory was launched on a full scale [2]. SSD is strong in shock and its I/O performance is better comparing with the conventional hard disk drive (HHD).

Though the storage rate of SSD increases remarkably compared to HHD, SSD alone is hard to meet most requirements of data acquisition and storage system $[3,4]$. Therefore, RAID 0 tech in computer science is introduced, and we propose an architecture of RAID, which is based on SSD and driven by FPGA, called SSD-based RAID on FPGA (short for SRF), which features mass capacity, high rate and small volume. In addition, in order to save costs, the architecture of SRF is designed to be adjustable in storage rate and capacity.

\section{Architecture of SSD-Based RAID on FPGA}

Figure 1 depicts the architecture of the SSD-based RAID on FPGA.

\subsection{SSD Controller}

SSD controllers, which directly determine whether the SSD-based RAID is good or bad, are the core of the architecture. Now, most SSD adopt SATAII with the line rate of 3.0 Gbps. Therefore, the core task is to design a SATA controller applying to SSD.

The physical connection of SATA could adopt the modules of speed serial interface given by FPGA manufacturers, such as ROCKET IO or Gbps transceivers. These modules with OOB signal for SATA physical layer have an amazing line rate, over $10 \mathrm{Gbps}$. In addition, there is the $8 \mathrm{~b} / 10 \mathrm{~b}$ coding in the modules to simplify the design of the SATA controller. In respect of clock processing, the modules differentiate the clock used by transceivers from the clock of the logic to avoid the occurrence of interference. There are more than 20 modules of speed serial interface in current top-end FPGA chips, and it's the precondition for building up the large RAID.

SATA data transmission adopts Frame Information Structure (FIS), and Figure 2 presents the DATA FIS. According to the protocol of SATA, a DATA FIS contains a maximum of 2048 Dwords, i.e. $8 \mathrm{kB}$ data. The method of transmission chooses the command of WRITE/READ DMA EXT through which SSD can read or write 4096 DATA FIS, i.e. $32 \mathrm{MB}$ data [5-7]. And the command of WRITE/READ DMA EXT can greatly reduce the time in writing or reading SSD.

In lower ATA versions, writing operation directly covers the garbage data. As for SSD, the garbage data need to be erased first, due to the features of the NAND flash memory, and it makes the writing rate lower. In 


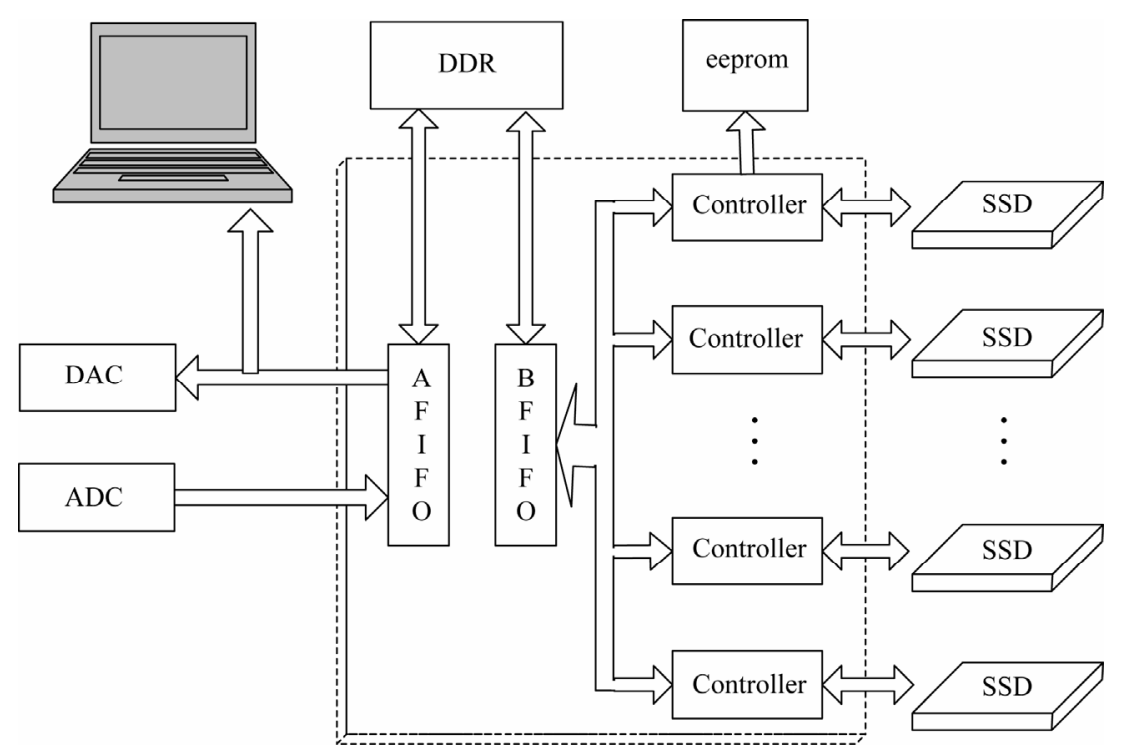

Figure 1. Architecture of the SSD-based RAID on FPGA.

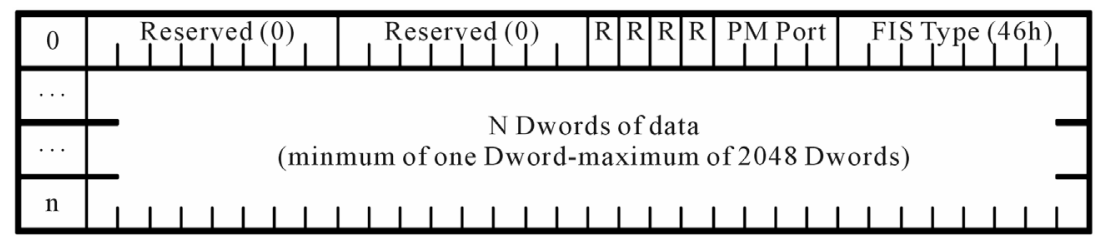

Figure 2. The data frame information structure.

order to accelerate the rate, TRIM command, which can erase the specific sector, is added to ATA8. In SSDbased RAID system, TRIM command should be executed before the writing operation.

Table 1 shows the test result of a demo SSD controller for a 256 GB SSD.

From the result, it can be seen that the writing rate of SSD drops as the data quantity grows, but still over 200 $\mathrm{MB} / \mathrm{s}$. The explanation for this, is that there is a buffer memory in SSD. The writing rate is limited by the buffer memory when the data quantity is small. As the data quantity grows, the writing rate declines. However, the reading rate of SSD isn't affected by the data quantity, and maintains at a level of, approximately, $224 \mathrm{MB} / \mathrm{s}$.

\subsection{RAID}

The multiple architecture of RAID is used for PC systems. This paper proposes an architecture of SSD-based RAID driven by a single FPGA.

As shown in Figure 3, the data stream is divided into several blocks, and the reading process goes in parallel with the writing process to accelerate the reading or writing rate. If an architecture driven by FPGA adopts this method, the way to improve the writing rate is to increase the quantity of SSD and accelerate the output rate of BFIFO in Figure 2. However, with the increasing
Table 1. The test result of a demo SSD controller.

\begin{tabular}{ccc}
\hline Data Quantity & Writing Rate $(\mathrm{MB} / \mathrm{s})$ & Reading Rate $(\mathrm{MB} / \mathrm{s})$ \\
\hline $32 \mathrm{MB}$ & 223 & 225 \\
$512 \mathrm{MB}$ & 218 & 223 \\
$2 \mathrm{~GB}$ & 201 & 223 \\
$48 \mathrm{~GB}$ & 200 & 225 \\
$250 \mathrm{~GB}$ & 201 & 224 \\
\hline
\end{tabular}

quantity of SSD, the number of fan-out of the BFIFO also increases. For high speed data stream of FPGA, having too much fan-out may cause the transmission error. In addition, the logic frequency is limited by the FPGA chip, so the output rate of BFIFO can't be accelerated without limits. Therefore, the method which divides the data stream is not applicable for RAID driven from FPGA.

Considering the features of FPGA, architecture of SSD-based RAID applies the method of dividing data bits to build up RAID. As shown in Figure 4, according to the high bits and low bits, data stream is divided into 4 groups data stream then written to SSD respectively. In this way, much fan-out of high speed data stream can be avoided, and the output of BFIFO in Figure 2 can be 


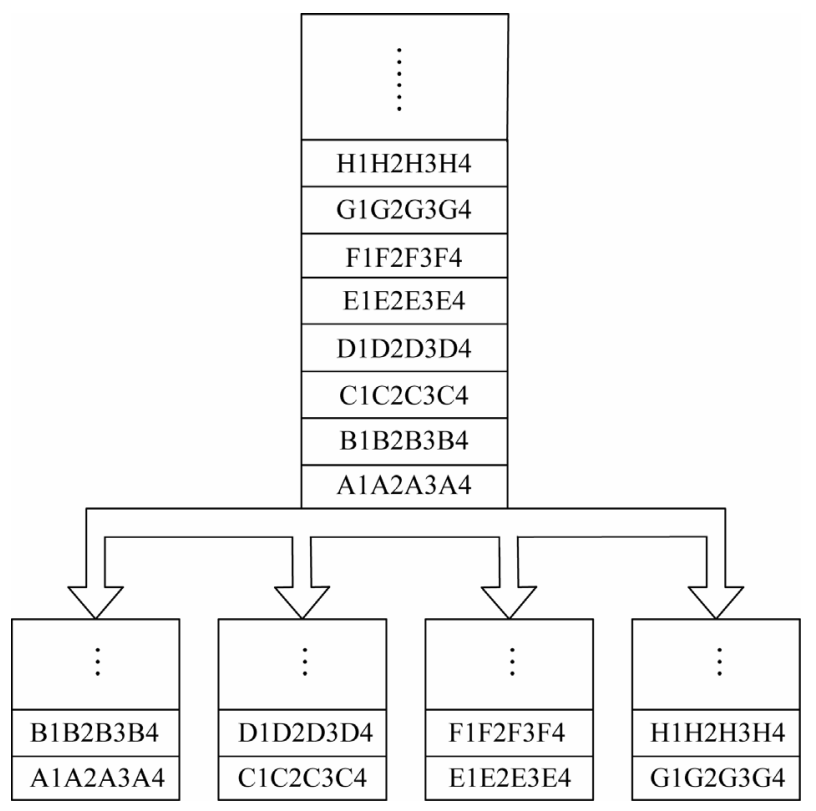

Figure 3. Data stream divided by data blocks.

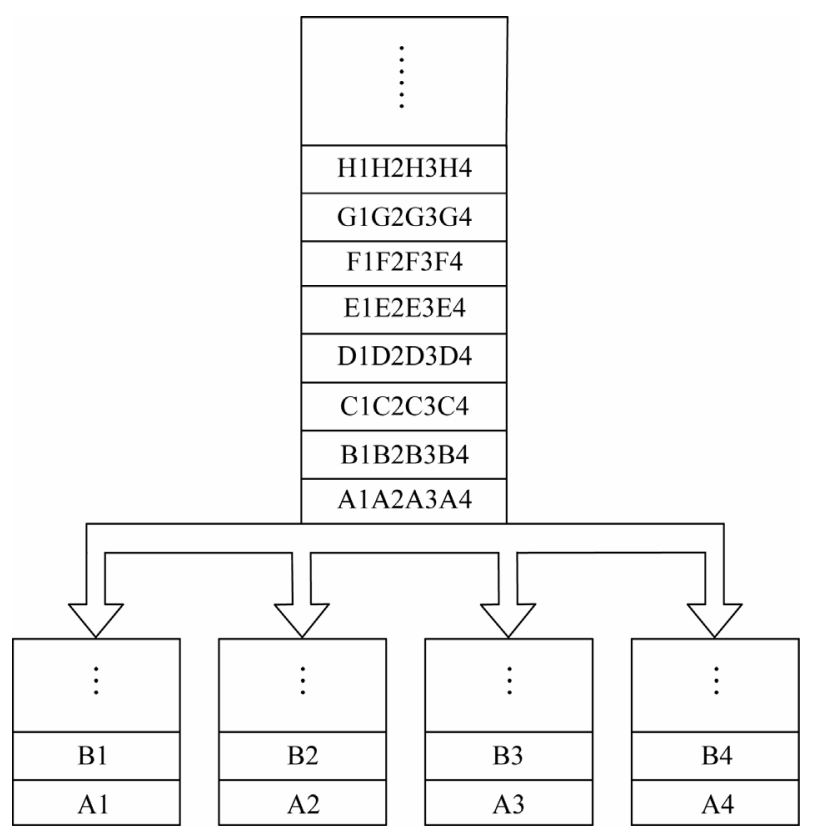

Figure 4. Data stream divided by data bits. kept at a steady rate, i.e. the interface rate of SATA. To accelerate the storage rate by dividing data bits, the width of BFIFO's output should be changed with the increasing quantity of SSD.

\subsection{Stream Data Buffer}

For the signal acquisition and storage system, to avoid the loss of data is the most important thing. However, the transmission of SATA contains redundant data, which vary in each SSD, and the interval between two FIS is hard to be in consistence. Admittedly, some SSD need to wait for others, and data stream has to stop as shown in Figure 5. To assure data integrity, a large capacity stream buffer is needed in architecture of SSD-based RAID. After selection, DDR chips are used as the stream buffer. Due to the time difference in reaching the $\mu$ s-grade, DDR, which usually has tens of MByte, is easy to buffer the data. The number of DDR is determined by the storage rate of the system. In the other words, the reading and writing rate of DDR is at least twice of the storage rate.

\subsection{Data Management}

Actually, the nature of the storage of acquired data is the storage of stream data. Theoretically, the storage information needs to record the original position and the quantity of the storage. There are two solutions to the problem of the storage information. One is to store the information in SSD with the acquired data. The other is to store the information in an extra memory chip such as EEPROM or flash memory. The latter is more suitable for the architecture of SSD-based RAID in following aspects. Firstly, when the information is stored in one $\mathrm{SSD}$, there is difference in RAID, which makes it hard for SSD of RAID to perform the unified operation. Secondly, because of the large capacity of SSD, to retrieve information is not an easy task. Thirdly, the stored information can be retrieved without RAID working if an extra memory chip is used. For these three reasons, the architecture should be used in storage of information.

The architecture should formulate the basic unit of storage. For example, the capacity of the storage system

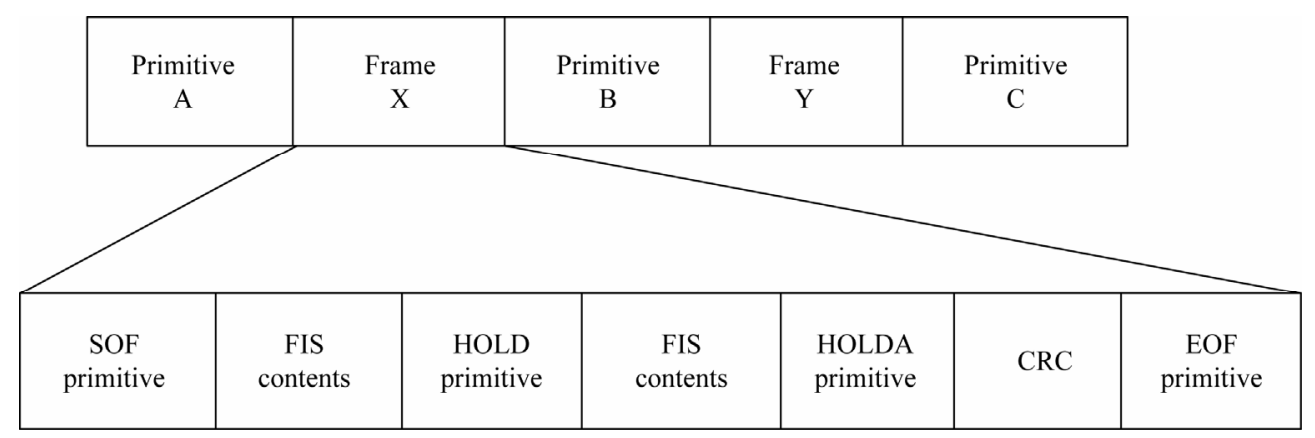

Figure 5. Transmission structures. 
is $100 \mathrm{~GB}$, and the user can set $1 \mathrm{~GB}$ as the basic unit. It is convenient for the data management system to divide the whole unit into 100 basic units.

\section{Test Result of a Demo SRF System}

\subsection{Test System and Environment}

In the test system, there are 18 SATA interface, building a RAID with maximum $18 \mathrm{SSD}$, and 2 DDR3 as the data buffer. In FPGA, a data generator, referring to the clock of $200 \mathrm{MHz}$, generates data stream with the width of 160-bits, i.e. the rate of $4 \mathrm{~GB} / \mathrm{s}$. The working of generator is controlled by the RAID system. When there is no room to buffer the data in RAID system, the generator is closed, and restarts when there is free space.

\subsection{Relationship between the Quantity of SSD and the Storage Rate}

The Table 2 shows the relationship between the quantity of SSD and the storage rate. 1) With the increasing quantity of SSD, the storage rate is raising monotonically. 2) The test rate of RAID system does not equal to the ideal rate (the ideal rate $=$ the quantity of SSD $*$ the rate of a single SSD). 3) The gap between the ideal rate and the test rate gets larger with the increasing quantity of SSD.

\subsection{Probability Distribution of Maximum Rate of RAID}

According to the results of the test, it can be found that maximum rate of RAID system isn't a constant. The Figure 6 respectively shows four probability distribution of maximum rate of the demo system with 2, 6, 12 and 18 SSD.

According to the results of the analysis, the gap between the test rate and the ideal rate is caused by the difference of each SSD. 1) It is uncertain for each SSD to have the same internal storage rate; 2) The transmission

Table 2. The test storage rate.

\begin{tabular}{cccc}
\hline $\begin{array}{c}\text { Quantity of } \\
\text { SSD }\end{array}$ & $\begin{array}{c}\text { Ideal storage rate } \\
(\mathrm{MB} / \mathrm{s})\end{array}$ & $\begin{array}{c}\text { Test storage rate } \\
(\mathrm{MB} / \mathrm{s})\end{array}$ & $\begin{array}{c}\text { Distance } \\
(\mathrm{MB} / \mathrm{s})\end{array}$ \\
\hline 1 & 200 & 200 & 0 \\
2 & 400 & 397 & 3 \\
4 & 800 & 792 & 8 \\
6 & 1200 & 1158 & 42 \\
8 & 1600 & 1557 & 43 \\
10 & 2000 & 1948 & 52 \\
12 & 2400 & 2338 & 62 \\
18 & 3600 & 3528 & 72 \\
\hline
\end{tabular}
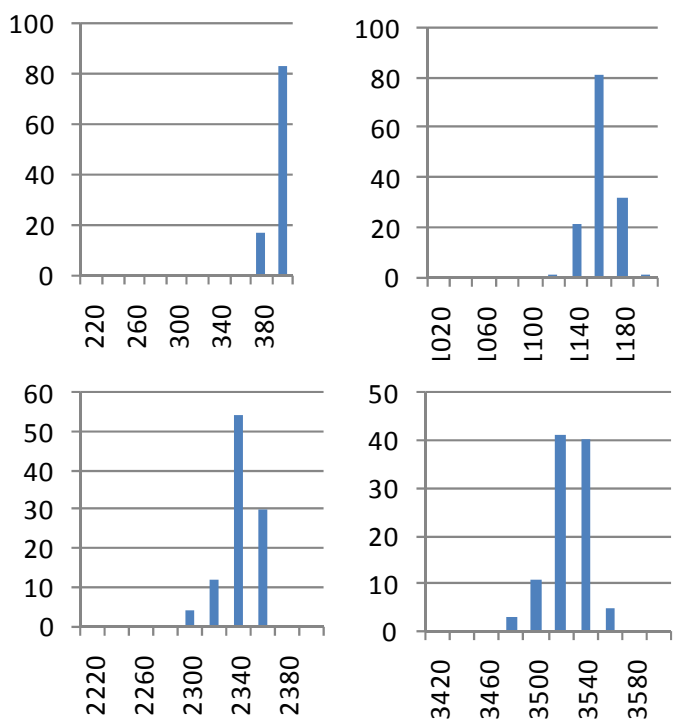

Figure 6. Probability distribution of maximum rate.

of SATA contains redundant data, and its length is also uncertain for each SSD; 3) Each SSD has different response time for the instruction. Based on the statement above, admittedly, some SSD has to wait for others. Hence the maximum rate of RAID system is reduced with the increasing quantity of SSD. But, apparently, the increase in number of SSD has the most predominant influence on the rate of RAID system.

\section{Conclusion and Discussion}

The test on demo system shows that the architecture of SRF has the ability to store high speed data stream and replay the storage data. Although the gap between the ideal rate and the test rate gets larger with the increasing quantity of SSD, it is considered to be within acceptable limits in engineering.

Our future work will focus on quantitative analysis to fit the function between the storage rate of SRF and the quantity of SSD, so that SRF will be applied conveniently. In the meantime, in order to improve the reliability, we will update the architecture of SRF based on RAID 4 or RAID 5. Despite the declining storage rate in the situation using RAID 4 or RAID 5 compared to RAID 0, the improvement of reliable is essential to engineering.

The architecture of SRF, which features mass capacity, high speed and small volume, will become an efficient solution to storage of broadband signal and high speed data in signal processing field.

\section{REFERENCES}

[1] K. Park, "Reliability and Performance Enhancement Technique for SSD Array Storage System Using RAID Mechanism," The 9th International Symposium on Com- 
munications and Information Technology (ISCIT 2009), 28-30 September 2009, pp. 140-145.

[2] W. F. Jones, "A Digital Architecture for Routinely Storing and Buffering the Entire 64-Bit Event Acquisition in Clinical Real-Time 3-D PET: Embedding a 400 Mbyte/ sec SATA RAID 0 Using a Set of Four Solid- Solid-State Drives," 2008 IEEE Nuclear Science Symposium Conference, 19-25 October 2008, pp. 5036-5040. doi:10.1109/NSSMIC.2008.4774371

[3] J. Ren, "I-CASH: Intelligently Coupled Array of SSD and HDD," The 17th International Symposium on High Performance Computer Architecture (HPCA), 12-16 February 2011, pp. 278-289.

[4] W. Wu, "Implementing a Serial ATA Controller base on FPGA," Computational Intelligence and Design (ISCID
09), Vol. 1, 12-14 December 2009, pp. 467-470.

[5] S. S. Rizvi, "Data Storage Framework on Flash Memory Based SSD RAID 0 for Performance Oriented Applications," The 2nd International Conference on Computer and Automation Engineering (ICCAE), Vol. 1, 26-28 February 2010, pp. 126-128.

[6] K. R. Dandekar, H. Ling and G. H. Xu, "Smart Antenna Array Calibration Procedure including Amplitude and Phase Mismatch and Mutual Coupling Effects," IEEE Conference on Personal Wireless Communications (PWC), Vol. 12, 2000, pp. 293-297.

[7] Serial ATA Working Group, "Serial ATA High Speed Serialized AT Attachment Rev 1.0a," Serial ATA Working Group, 2002, pp. 117-123. 